\title{
Quantum Mechanics and Narratability
}

\author{
Wayne C. Myrvold \\ Department of Philosophy \\ The University of Western Ontario \\ London, ON Canada N6A 5B8 \\ wmyrvold@uwo.ca \\ Forthcoming in Foundations of Physics
}

\begin{abstract}
As has been noted by several authors, in a relativistic context, there is an interesting difference between classical and quantum state evolution. For a classical system, a state history of a quantum system given along one foliation uniquely determines, without any consideration of the system's dynamics, a state history along any other foliation. This is not true for quantum state evolution; there are cases in which a state history along one foliation is compatible with multiple distinct state histories along some other, a phenomenon that David Albert has dubbed "non-narratability." In this article, we address the question of whether non-narratability is restricted to the sorts of special states that so far have been used to illustrate it. The results of the investigation suggest that there has been a misplaced emphasis on underdetermination of state histories; though this is generic for the special cases that have up until now been considered, involving bipartite systems in pure entangled states, it fails generically in cases in which more component systems are taken into account, and for bipartite systems that have some entanglement with their environment. For such cases, if we impose relativistic causality constraints on the evolution, then, except for very special states, a state history along one foliation uniquely determines a state history along any other. But this in itself is a marked difference between classical and quantum state evolution, because, in a classical setting, no considerations of dynamics at all are needed to go from a state history along one foliation to a state history along another. Keywords: relativistic quantum theory, narratability, nonseparability
\end{abstract}




\section{Introduction}

As has been noted by several authors (Aharonov and Albert (1984), Myrvold (2002), Albert and Galchen (2009), Albert (2015)), there is an interesting difference between state evolution of classical and quantum systems in relativistic setting. For a classical system, one can provide a state history specifying a complete state of a system on each of a set of spacelike hypersurfaces that foliate the time interval of interest, and this yields, without further ado, and without considerations of dynamics, a state history along any other foliation. This is because classical states satisfy the condition of separability: to completely specify the state of a system, it suffices to specify the states of things in arbitrarily small regions of space, and so one can construct a state on any hypersurfaces out of states on hypersurfaces that intersect it.

With quantum systems, things are different. The state of a composite system composed of spatially separated entangled parts is not determined by the states of its component parts. This means that the piecewise construction of a state on one hypersurface out of bits of states on hypersurfaces that intersect it will not work. As a consequence, consideration of the dynamics of the system is required when passing from a state history along one foliation to another.

Though noted before, this difference between classical and quantum state evolution has been characterized in a potentially misleading way. It has been illustrated by examples of a pair of systems in a pure entangled state, having the property that a state evolution along one foliation is compatible with multiple distinct evolutions along other foliations, even when relativistic causality constraints are taken into account, a phenomenon that Albert Albert (2015) has called "nonnarratability." Though this phenomenon is (as we shall see, below) generic for bipartite systems in entangled states, once we consider systems composed of more than two spatially separated components, it holds only for very special states. For systems composed of three or more spatially separated parts (which could include bipartite systems that are to some degree entangled with the environment), for typical states, a state history along one foliation together with the condition that the dynamics of the system obey relativistic causality constraints yields enough information about the dynamical evolution of the system to uniquely determine a state history along any other foliation.

Thus, it is a bit misleading to characterize the difference between relativistic state evolution of classical and quantum systems the way it has been up to now, in terms of whether or not a state evolution along one foliation uniquely determines state histories along others. If we impose relativistic causality constraints on the 
evolution, then, except for very special states, a state history along one foliation uniquely determines a state history along any other. But this in itself is a marked difference between classical and quantum state evolution, because, in a classical setting, no considerations of dynamics at all are needed to go from a state history along one foliation to a state history along another.

\section{The set-up}

We consider a finite number of quantum systems located in regions of space distant from each other. Between time $t_{0}$ and $t_{1}$ (as given by the time coordinate of some reference frame) each undergoes some evolution. One can imagine agents at each location choosing between operations to perform on the systems.

Suppose, now, that you are not told what evolution each system individually undergoes, but you are given the state of the composite system at each time during the time interval from $t_{0}$ to $t_{1}$. Suppose that the systems are far enough apart, and the time interval from $t_{0}$ to $t_{1}$ short enough, that the systems are spacelike separated from each other throughout the interval. Then, one can choose a spacelike hypersurface that coincides with $t_{0}$ at the location of some of the systems and $t_{1}$ at others, and, taking this as a hypersurface of simultaneity, ask for the quantum state on it. We ask: does the state history you were originally given permit you to infer the state of the system on such a hypersurface of simultaneity?

The question amounts to the question of whether knowing the net effect of component operations on the initial state of the combined system permits one to infer the effect of each taken singly. If we consider a bipartite system in a pure state, the answer is that no, it does not; it is easy to construct examples in which a composite system $A B$ is in a pure entangled state and the net effect of the pair of operations on the composite system underdetermines the effect of the operations taken singly. Thus, within the idealizations of the set-up described, a state history of the composite system given along one foliation might not, by itselfthat is, without specification of the laws governing the dynamical evolution of the systems-determine the state history along another; this is what Albert calls "non-narratability" Albert (2015). It has thus far been illustrated by simple examples that, for ease of exposition, are chosen to have very special features. These examples involve pure states of bipartite systems, evolving in isolation from their environments; moreover, they also involve maximally entangled states. One might wonder whether the phenomenon is restricted to special states such as those used in the examples_-which would, perhaps, diminish the interest of such examples- 
or whether it holds more generally. In this article we present some results in connection with this question. For simplicity, in this article we restrict ourselves to considerations of systems confined to regions that are small compared to the distances between them, so that relativistic considerations other than relativity of simultaneity may be ignored. The question of the extension of these results to fully relativistic quantum theories, which must be quantum field theories, will be considered in a sequel.

These results may be summarized as follows:

1. For the sorts of examples used to illustrate non-narratability, in which a bipartite system is taken to be in a pure state (not entangled with its environment) and to undergo unitary evolution, non-narratability prevails. For any entangled initial state-vector $|\psi(\alpha)\rangle$ and any state-vector $|\psi(\beta)\rangle$ obtained from $|\psi(\alpha)\rangle$ by a factorizable unitary evolution $U_{A} \otimes U_{B}$, there will exist other unitaries $V_{A}, V_{B}$, such that

$$
|\psi(\beta)\rangle=U_{A} \otimes U_{B}|\psi(\alpha)\rangle=V_{A} \otimes V_{B}|\psi(\alpha)\rangle,
$$

but

$$
U_{A} \otimes I|\psi(\alpha)\rangle \neq V_{A} \otimes I|\psi(\alpha)\rangle \text {. }
$$

2. For a bipartite system initially in a pure state, non-narratability persists, for a wide class of states and evolutions, if the condition of isolated evolution is relaxed.

3. For systems of more than two parts, whose parts separately undergo unitary evolution, we have the opposite result: generically, the state history of the system along one foliation uniquely determines its state history along any other, given the condition (required by relativistic causality) that the total evolution operator is a product of commuting evolution operators for the component systems.

4. It follows that the same holds for a bipartite system not required to be initially in a pure state, but which may be entangled with its environment, whose parts undergo unitary evolution. Generically, the state history of the system along one foliation uniquely determines its state history along any other.

Now, even for for a bipartite system, isolated from its environment, one should expect some entanglement with the environment. Then, for generic states, nonnarratability fails. 


\section{Background}

The "non-narratability" phenomenon was noted in work by Aharonov and Albert in the mid-1980s. Aharonov and Albert Aharonov and Albert (1984) discuss a set-up in which a singlet spin state of a pair of spin- $\frac{1}{2}$ particles is 'verified' by experimental apparatus, in the following sense: if the system is in the singlet state, it remains in the single state, and the experimental apparatus registers this fact; if the system is not initially in the singlet state, then its state is disturbed by its interaction with the apparatus (this can be done via local interactions with each of the particles, if they are spatially separated, though it requires nonlocal entanglement of the experimental apparatus). In the experimental scheme outlined by Aharonov and Albert, the condition that the experiment leave the singlet state undisturbed requires the local couplings of each of the particles with the apparatus to be synchronized: the interactions between the particles and their experimental apparatus must be switched on simultaneously. Simultaneity is, of course, not a frame-independent notion, so the interaction is non-disturbing only with respect to one particular reference frame; with respect to others, it first disturbs the state, and then restores it. As was pointed out by Myrvold Myrvold (2002), this holds also outside of measurement situations. The example adduced therein involved a pair of spin- $\frac{1}{2}$ particles, initially in the singlet state. Identical magnetic fields are switched on at both wings of the system, inducing spin precessions in opposite directions that-provided that the switching is simultaneous-cancel each other out, leaving the state unchanged.

David Albert Albert (2015) discusses an example involving four spin- $\frac{1}{2}$ particles, all having inertial trajectories, such that the trajectory of particle 3 intersects that of particle 1 , the trajectory of particle 4 intersects that of particle 2 , and these two intersections happen at spacelike separation. Initially, particles 1 and 2 are in a singlet state, as are particles 3 and 4 . At the moments of intersection, the particles involve undergo an interaction that swaps their spins. With respect to a foliation on which the two swaps are simultaneous, the spin states remain forever unchanged. With respect to a foliation that has the 1-3 swap before the 2-4 swap, the system is for some time in a state in which particle 1 is entangled with particle 4 , and particle 2 with particle 3 , before the second swap restores the initial state. Once again, a complete state history along one foliation underdetermines the state history along other foliations. The state history with simultaneous swaps, on which the spin state of the combined system remains forever constant, is of course the same state history that would obtain in the absence of the swap interaction. But these two scenarios yield different state histories along foliations 
on which the two intersections are not simultaneous.

Non-narratability has been described as a "quantum threat to special relativity" Albert and Galchen (2009). In connection with this, the authors write,

combining quantum mechanics and special relativity requires that we give up another of our primordial convictions. We believe that everything there is to say about the world can in principle be put into the form of a narrative, or story. Or, in more precise and technical terms: everything there is to say can be packed into an infinite set of propositions of the form "at $t 1$ this is the exact physical condition of the world" and "at $t 2$ that is the exact physical condition of the world," and so on. But the phenomenon of quantum-mechanical entanglement and the spacetime geometry of special relativity-taken together-imply that the physical history of the world is infinitely too rich for that (Albert and Galchen, 2009, p. 39).

It is a bit misleading to say that the lesson to be learned is that we have to give the idea that everything there is to say about the world can in principle be put into the form of a narrative. What the examples show is that a narrative of a certain kind, namely, a narrative that consists merely of a compendium of descriptions of instantaneous states according to one reference frame's hyperplanes of simultaneity, and says nothing about the dynamics that lead from one state to another, is not a complete story. To say everything that there is to say about, say, a pair of spin- $\frac{1}{2}$ particles during a certain time interval, it is not enough to say that their state with respect to a certain foliation is a singlet state at all times in that interval; one must also specify that this is (or isn't) due to spin precessions in opposite directions that cancel each other out.

As we shall see, it is only for certain special states that we have to specify even that much. For generic states, all that need be done is to specify that the evolution is via some evolutions that respect relativistic causality, and this will uniquely specify the state history. But, even when this fact is taken into account, there remains an essential difference between classical and quantum state histories: for classical states, the transition from a state history along one foliation to a state history along another requires no considerations of dynamics at all. 


\section{QM in a quasi-relativistic setting}

We consider a finite number of quantum systems localized in bounded regions of space. Let $\left\{s_{i}\right\}$ be the world-tubes of these regions, let $\alpha$ be a spacelike hypersurface, and let $\beta$ be a spacelike hypersurface to the future of $\alpha$, such that, for each $s_{i}, s_{i}(\alpha)=s_{i} \cap \alpha$ is spacelike separated from $s_{j}(\beta)$ for all $j \neq i$.

Because we are considering state evolution, it is convenient to operate with a Schrödinger picture, adapted to a relativistic setting. Thus, we associate, timelessly, with each system an algebra $\mathscr{A}_{i}$, whose self-adjoint elements represent observables on the system. These algebras commute for distinct $i, j$. Let $\mathscr{A}$ be the smallest algebra containing each $\mathscr{A}_{i}$.

$$
\mathscr{A}=\bigvee_{i} \mathscr{A}_{i}
$$

The state of a system on a given hypersurface $\sigma$ will be given by a density operator $\rho(\sigma)$; this yields probabilities for outcomes of experiments performed on $\sigma$. If the system is in a pure state, that is, not entangled with anything outside of it, we will also be able to represent its state by a state vector.

If the systems are isolated from their environments during the interval between $\alpha$ and $\beta$, or if they are subject only to external fields that can be treated classically (and if there is no collapse), there will be unitary operators $U_{i}(\beta ; \alpha)$ such that the evolution of the combined system from $\alpha$ to $\beta$ is given by

$$
U(\beta ; \alpha)=\prod_{i} U_{i}(\beta ; \alpha)
$$

Consider a spacelike hypersurface $\gamma$ that includes $s_{i} \cap \alpha$ for some $i$, and $s_{i} \cap \beta$ for others (we can smoothly interpolate in between, but these regions will be of no concern). Then $U_{i}(\gamma ; \alpha)$ will be equal to $U_{i}(\beta ; \alpha)$ when the intersection of $s_{i}$ with $\gamma$ is in $\beta$, and to the identity when this intersection is in $\alpha$.

If the systems are not isolated from their respective environments, then the states of the systems on the later hypersurface $\beta$ will not be determined by their states on the earlier hypersurface, $\alpha$. In such a case we consider the casual pasts on $\alpha$ of the regions $s_{i}(\beta)$; call these $\tilde{s}_{i}(\alpha)$. We will assume that these are disjoint for distinct $i, j$. Associated with such regions will be algebras $\tilde{\mathscr{A}}_{i}$, with $\mathscr{A}_{i} \subseteq \tilde{\mathscr{A}}_{i}$. Let $\tilde{\mathscr{A}}=\mathrm{V}_{i} \tilde{\mathscr{A}}$.

Let the state of $\cup_{i} \tilde{s}_{i}(\alpha)$ be given by a density operator $\tilde{\rho}(\alpha)$, and the state of $\cup_{i} s_{i}(\beta)$, by $\rho(\beta)$. There there will be a factorizable unitary operator $U(\beta ; \alpha)$ such that

$$
\operatorname{Tr}[\rho(\beta) A]=\operatorname{Tr}\left[U^{\dagger}(\beta ; \alpha) \tilde{\rho}(\alpha) U(\beta ; \alpha) A\right]
$$


for any $A \in \mathscr{A}$. This defines a mapping $\tilde{\rho}(\alpha) \rightarrow \rho(\beta)$. Note that, since $\mathscr{A}$ is a proper subset of $\tilde{\mathscr{A}}$, this mapping can take a pure state $\tilde{\rho}(\alpha)$ into a mixed state $\rho(\beta)$.

\section{Results concerning narratability and non-narratability}

\subsection{Bipartite systems with pure initial states: non-narratability is generic}

We consider first the case of two systems, with world-tubes $s_{A}$ and $s_{B}$, with which we associate operator algebras $\mathscr{A}$ and $\mathscr{B}$. Suppose we know the state of the combined system on $\alpha$ and $\beta$. Let $\gamma$ be a spacelike hypersurface containing $s_{A}(\beta)$ and $s_{B}(\alpha)$, and let $\delta$ contain $s_{A}(\alpha)$ and $s_{B}(\beta)$ (see Figure 1). The question to be asked is: what do the initial and final states $\rho_{A B}(\alpha), \rho_{A B}(\beta)$ tell us about the evolution in between, and hence about $\rho_{A B}(\gamma)$ and $\rho_{A B}(\delta)$ ?

We will make use of the fact that any vector $|\psi\rangle$ in $\mathscr{H}_{A} \otimes \mathscr{H}_{B}$ can be written in the form,

$$
|\psi\rangle=\sum_{k} c_{k}\left|a_{k}\right\rangle \otimes\left|b_{k}\right\rangle
$$

where $\left\langle a_{n} \mid a_{m}\right\rangle=\left\langle b_{n} \mid b_{m}\right\rangle=\delta_{m n}$. Such a representation is called a Schmidt representation. In the non-degenerate case-that is, $\left|c_{i}\right| \neq\left|c_{j}\right|$ for $i \neq j$-the elements of the sets $\left\{\left|a_{k}\right\rangle\right\}$ and $\left\{\left|b_{k}\right\rangle\right\}$ are determined up to phase factors. In the degenerate case, there is more leeway. It will turn out that, in the case in which the state of $A B$ is pure on both $\alpha$ and $\beta$, the scope of underdetermination of $|\psi(\gamma)\rangle$ and $\mid \psi(\delta\rangle)$ by $|\psi(\alpha)\rangle$ and $|\psi(\beta)\rangle$ is related in a simple way to the scope of underdetermination of the Schmidt representations of $|\psi(\alpha)\rangle$ and $|\psi(\beta)\rangle$.

\subsubsection{Unitary evolution}

Consider first the case in which the systems are isolated or subject only to external fields that can be treated classically. Then there are unitary operators $U_{A}, U_{B}$, such that

$$
\begin{aligned}
|\psi(\beta)\rangle & =U_{A} \otimes U_{B}|\psi(\alpha)\rangle \\
|\psi(\gamma)\rangle & =U_{A} \otimes I|\psi(\alpha)\rangle \\
|\psi(\delta)\rangle & =I \otimes U_{B}|\psi(\alpha)\rangle .
\end{aligned}
$$




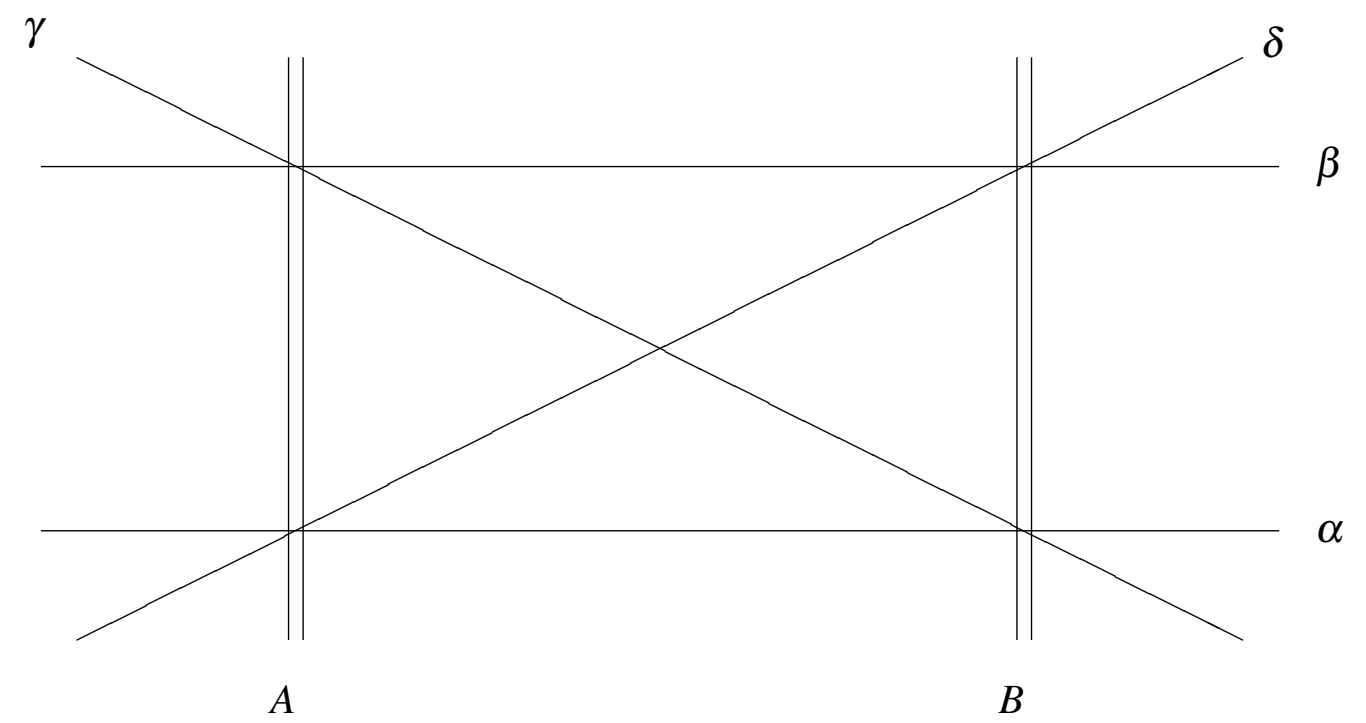

Figure 1: The hyperplanes considered in the proof.

Zurek Zurek (2005) has shown that, for any bipartite system $A B$ in a pure entangled state, there are unitary operations that can be performed on $A$ that change the state that can be undone by a unitary operation performed on $B$. In Zurek's terminology, a state of a bipartite system $A B$ is envariant with respect to a transformation acting on $A$ if it can be undone by a transformation acting on $B$. Zurek proved that the transformations under which a state is envariant are precisely those whose eigenstates are a Schmidt basis.

Now, if a state $|\psi(\beta)\rangle$ can be obtained in one of two ways from $|\psi(\alpha)\rangle$; that is, if

$$
|\psi(\beta)\rangle=U_{A} \otimes U_{B}|\psi(\alpha)\rangle=V_{A} \otimes V_{B}|\psi(\alpha)\rangle,
$$

then $|\psi(\alpha)\rangle$ is invariant under the transformation

$$
W=W_{A} \otimes W_{B}=U_{A}^{\dagger} V_{A} \otimes U_{B}^{\dagger} V_{B} .
$$

The state is, therefore, envariant with respect to the transformation $W_{A}$ on $A$. Moreover, if $|\psi(\alpha)\rangle$ is invariant under a transformation $W_{A} \otimes W_{B}$, such that $|\psi(\alpha)\rangle$ 
is not invariant under $W_{A}$ or $W_{B}$ alone, then, given for any evolution $U_{A} \otimes U_{B}$ that takes $|\psi(\alpha)\rangle$ into $|\psi(\beta)\rangle, U_{A} W_{A} \otimes U_{B} W_{B}$ is another evolution that has the same effect on $|\psi(\alpha)\rangle$. Therefore, Zurek's theorem on envariance gives us a complete characterization of the possible intermediate states $|\psi(\gamma)\rangle$ and $|\psi(\delta)\rangle$.

Given $|\psi(\alpha)\rangle$ and $|\psi(\beta)\rangle$, related by some factorizable unitary transformation, here's the recipe for generating possible states $|\psi(\gamma)\rangle$ and $|\psi(\delta)\rangle$ on the intermediate hypersurfaces. Take any Schmidt representations for $|\psi(\alpha)\rangle$ and $|\psi(\beta)\rangle$, having the same coefficients $\left\{c_{k}\right\}$ (this will always be possible in the case considered, of factorizable unitary evolution),

$$
\begin{aligned}
|\psi(\alpha)\rangle & =\sum_{k} c_{k}\left|a_{k}\right\rangle \otimes\left|b_{k}\right\rangle \\
|\psi(\beta)\rangle & =\sum_{k} c_{k}\left|a_{k}^{\prime}\right\rangle \otimes\left|b_{k}^{\prime}\right\rangle .
\end{aligned}
$$

Then the following are candidates for states on $\gamma$ and $\delta$ :

$$
\begin{aligned}
|\psi(\gamma)\rangle & =\sum_{k} c_{k}\left|a_{k}^{\prime}\right\rangle \otimes\left|b_{k}\right\rangle \\
|\psi(\delta)\rangle & =\sum_{k} c_{k}\left|a_{k}\right\rangle \otimes\left|b_{k}^{\prime}\right\rangle
\end{aligned}
$$

Moreover, any possible pair of states on $\gamma$ and $\delta$ can be generated in this way from Schmidt representations of $|\psi(\alpha)\rangle$ and $|\psi(\beta)\rangle$.

As an example, suppose that the evolution from $\alpha$ to $\beta$ leaves the state unchanged,

$$
|\psi(\alpha)\rangle=|\psi(\beta)\rangle=\sum_{k} c_{k}\left|a_{k}\right\rangle \otimes\left|b_{k}\right\rangle
$$

and suppose that that this is a case of non-degeneracy, $\left|c_{i}\right| \neq\left|c_{j}\right|$ for $i \neq j$. Then, since the Schmidt representation is unique up to phase factors, there must exist a set $\left\{\theta_{k}\right\}$ of real numbers such that

$$
\begin{aligned}
|\psi(\gamma)\rangle & =\sum_{k} c_{k} e^{i \theta_{k}}\left|a_{k}\right\rangle \otimes\left|b_{k}\right\rangle \\
|\psi(\delta)\rangle & =\sum_{k} c_{k} e^{-i \theta_{k}}\left|a_{k}\right\rangle \otimes\left|b_{k}\right\rangle
\end{aligned}
$$

So, in the nondegenerate case, what is underdetermined is the relative phases of the terms of the Schmidt representations of $|\psi(\gamma)\rangle$ and $|\psi(\delta)\rangle$. 
In the degenerate case, the states on $\gamma$ and $\delta$ will be generated from some Schmidt decomposition of the state on $\alpha$ and $\beta$. (This is the case under which Albert's example falls; see below.)

We now establish the claim that the recipe works, and generates all possibilities for states on the intermediate hypersurfaces $\gamma$ and $\delta$ (this is an application of Zurek's theorem to the question at hand).

Proposition 1 a). Suppose that $|\psi(\alpha)\rangle,|\psi(\beta)\rangle \in \mathscr{H}_{A} \otimes \mathscr{H}_{B}$ are related by some factorizable unitary transformation,

$$
|\psi(\beta)\rangle=U_{A} \otimes U_{B}|\psi(\alpha)\rangle,
$$

Take any Schmidt representations of $|\psi(\alpha)\rangle$ and $|\psi(\beta)\rangle$ sharing the same coefficients,

$$
\begin{aligned}
|\psi(\alpha)\rangle & =\sum_{k} c_{k}\left|a_{k}\right\rangle \otimes\left|b_{k}\right\rangle \\
|\psi(\beta)\rangle & =\sum_{k} c_{k}\left|a_{k}^{\prime}\right\rangle \otimes\left|b_{k}^{\prime}\right\rangle,
\end{aligned}
$$

and define

$$
\begin{aligned}
|\psi(\gamma)\rangle & =\sum_{k} c_{k}\left|a_{k}^{\prime}\right\rangle \otimes\left|b_{k}\right\rangle \\
|\psi(\delta)\rangle & =\sum_{k} c_{k}\left|a_{k}\right\rangle \otimes\left|b_{k}^{\prime}\right\rangle .
\end{aligned}
$$

Then there exist unitary $V_{A}, V_{B}$ such that

$$
\begin{aligned}
|\psi(\beta)\rangle & =V_{A} \otimes V_{B}|\psi(\alpha)\rangle \\
|\psi(\gamma)\rangle & =V_{A} \otimes I|\psi(\alpha)\rangle \\
|\psi(\delta)\rangle & =I \otimes V_{B}|\psi(\alpha)\rangle .
\end{aligned}
$$

b). Suppose that $|\psi(\alpha)\rangle,|\psi(\beta)\rangle|\psi(\gamma)\rangle$, and $|\psi(\delta)\rangle$ are vectors in $\mathscr{H}_{A} \otimes \mathscr{H}_{B}$ that are related by

$$
\begin{aligned}
|\psi(\beta)\rangle & =U_{A} \otimes U_{B}|\psi(\alpha)\rangle \\
|\psi(\gamma)\rangle & =U_{A} \otimes I|\psi(\alpha)\rangle \\
|\psi(\delta)\rangle & =I \otimes U_{B}|\psi(\alpha)\rangle,
\end{aligned}
$$


for some unitary $U_{A}, U_{B}$. Then there exist Schmidt representations of $|\psi(\alpha)\rangle$ and $|\psi(\beta)\rangle$,

$$
\begin{aligned}
|\psi(\alpha)\rangle & =\sum_{k} c_{k}\left|a_{k}\right\rangle \otimes\left|b_{k}\right\rangle \\
|\psi(\beta)\rangle & =\sum_{k} c_{k}\left|a_{k}^{\prime}\right\rangle \otimes\left|b_{k}^{\prime}\right\rangle
\end{aligned}
$$

such that

$$
\begin{aligned}
|\psi(\gamma)\rangle & =\sum_{k} c_{k}\left|a_{k}^{\prime}\right\rangle \otimes\left|b_{k}\right\rangle \\
|\psi(\delta)\rangle & =\sum_{k} c_{k}\left|a_{k}\right\rangle \otimes\left|b_{k}^{\prime}\right\rangle .
\end{aligned}
$$

Proof 1 a) Let $|\psi(\alpha)\rangle,|\psi(\beta)\rangle$ be related by some factorizable unitary transformation. Then there exists at least one pair of Schmidt representations for these two vectors having the same coefficients. Take any such pair,

$$
\begin{aligned}
|\psi(\alpha)\rangle & =\sum_{k} c_{k}\left|a_{k}\right\rangle \otimes\left|b_{k}\right\rangle \\
|\psi(\beta)\rangle & =\sum_{k} c_{k}\left|a_{k}^{\prime}\right\rangle \otimes\left|b_{k}^{\prime}\right\rangle,
\end{aligned}
$$

and define

$$
\begin{aligned}
|\psi(\gamma)\rangle & =\sum_{k} c_{k}\left|a_{k}^{\prime}\right\rangle \otimes\left|b_{k}\right\rangle \\
|\psi(\delta)\rangle & =\sum_{k} c_{k}\left|a_{k}\right\rangle \otimes\left|b_{k}^{\prime}\right\rangle .
\end{aligned}
$$

If the sets $\left\{\left|a_{k}\right\rangle\right\},\left\{\left|b_{k}\right\rangle\right\},\left\{\left|a_{k}^{\prime}\right\rangle\right\},\left\{\left|b_{k}^{\prime}\right\rangle\right\}$ fail to span their respective spaces, they can be extended to orthonormal sets that do. Define

$$
\begin{aligned}
V_{A} & =\sum_{k}\left|a_{k}^{\prime}\right\rangle\left\langle a_{k}\right| \\
V_{B} & =\sum_{k}\left|b_{k}^{\prime}\right\rangle\left\langle b_{k}\right|,
\end{aligned}
$$

where the sums are taken over the orthonormal bases that include the Schmidt vectors we started with. These are unitary operators having the desired properties. 
b) Suppose that $|\psi(\alpha)\rangle$, $|\psi(\beta)\rangle|\psi(\gamma)\rangle$, and $|\psi(\delta)\rangle$ are vectors in $\mathscr{H}_{A} \otimes \mathscr{H}_{B}$ that are related by

$$
\begin{aligned}
|\psi(\beta)\rangle & =U_{A} \otimes U_{B}|\psi(\alpha)\rangle \\
|\psi(\gamma)\rangle & =U_{A} \otimes I|\psi(\alpha)\rangle \\
|\psi(\delta)\rangle & =I \otimes U_{B}|\psi(\alpha)\rangle,
\end{aligned}
$$

for some unitary $U_{A}, U_{B}$. Take any Schmidt representation of $|\psi(\alpha)\rangle$,

$$
|\psi(\alpha)\rangle=\sum c_{k}\left|a_{k}\right\rangle \otimes\left|b_{k}\right\rangle
$$

and define

$$
\begin{aligned}
& \left|a_{k}^{\prime}\right\rangle=U_{A}\left|a_{k}\right\rangle \\
& \left|b_{k}^{\prime}\right\rangle=U_{B}\left|b_{k}\right\rangle .
\end{aligned}
$$

Then, clearly $\left\{\left|a_{k}^{\prime}\right\rangle\right\}$ and $\left\{\left|b_{k}^{\prime}\right\rangle\right\}$ are orthonormal sets, and

$$
|\psi(\beta)\rangle=\sum c_{k}\left|a_{k}^{\prime}\right\rangle \otimes\left|b_{k}^{\prime}\right\rangle
$$

The sets $\left\{\left|a_{k}^{\prime}\right\rangle\right\}$ and $\left\{\left|b_{k}^{\prime}\right\rangle\right\}$, therefore, are a Schmidt representation of $|\psi(\beta)\rangle$. Moreover,

$$
\begin{aligned}
|\psi(\gamma)\rangle & =\sum_{k} c_{k}\left|a_{k}^{\prime}\right\rangle \otimes\left|b_{k}\right\rangle \\
|\psi(\delta)\rangle & =\sum_{k} c_{k}\left|a_{k}\right\rangle \otimes\left|b_{k}^{\prime}\right\rangle
\end{aligned}
$$

as required.

We thus have non-narratability, not only for special initial states, but for any entangled state. The only initial states for which the recipe will fail to generate a multiplicity of candidates for the states $|\psi(\gamma)\rangle$ and $|\psi(\delta)\rangle$ will be factorizable initial states.

If we take the Haar measure on the space of pure states of the bipartite system, the set of factorizable states is a set of measure zero. Moreover, on the norm topology, the set of entangled states is an open set that is dense in $\mathscr{H}_{A} \otimes \mathscr{H}_{B}$ : every neighborhood of a factorizable state contains entangled states, though every entangled state has a neighborhood consisting entirely of entangled states. In this sense, entanglement is a generic feature of states, and so, non-narratibility (at least when the initial state of the bipartite system is pure) is a generic feature. 
We illustrate the theorem with some simple examples.

Example 1. Spin precession. Consider a pair of spin- $\frac{1}{2}$ particles, initially in the singlet state:

$$
\left|\Psi^{-}\right\rangle=\frac{1}{\sqrt{2}}\left(\left|z^{+}\right\rangle\left|z^{-}\right\rangle-\left|z^{-}\right\rangle\left|z^{+}\right\rangle\right)
$$

where $\left|z^{+}\right\rangle$and $\left|z^{-}\right\rangle$are spin-up and spin-down in the $\hat{z}$-direction. Equation (4) gives one Schmidt representation of the state. If, however, we consider any other direction, say, $\hat{m}$, we have another Schmidt representation,

$$
\left|\Psi^{-}\right\rangle=\frac{1}{\sqrt{2}}\left(\left|m^{+}\right\rangle\left|m^{-}\right\rangle-\left|m^{-}\right\rangle\left|m^{+}\right\rangle\right)
$$

Take $U_{1}$ and $U_{2}$ to be the same rotations on each side,

$$
U_{i}\left|m^{+}\right\rangle=\left|n^{+}\right\rangle \quad U_{i}\left|m^{-}\right\rangle=\left|n^{-}\right\rangle .
$$

Then $U_{1} \otimes U_{2}$ leaves the state unchanged.

$$
\begin{aligned}
|\psi(\beta)\rangle & =U_{1} \otimes U_{2}\left|\Psi^{-}\right\rangle=\frac{1}{\sqrt{2}}\left(U_{1}\left|m^{+}\right\rangle \otimes U_{2}\left|m^{-}\right\rangle-U_{1}\left|m^{-}\right\rangle \otimes U_{2}|m+\rangle\right) \\
& =\frac{1}{\sqrt{2}}\left(\left|n^{+}\right\rangle\left|n^{-}\right\rangle-\left|n^{-}\right\rangle\left|n^{+}\right\rangle\right)=\left|\Psi^{-}\right\rangle .
\end{aligned}
$$

The states on the intermediate hypersurfaces will be given by,

$$
\begin{aligned}
& |\psi(\gamma)\rangle=\frac{1}{\sqrt{2}}\left(\left|n^{+}\right\rangle\left|m^{-}\right\rangle-\left|n^{-}\right\rangle\left|m^{+}\right\rangle\right) \\
& |\psi(\delta)\rangle=\frac{1}{\sqrt{2}}\left(\left|m^{+}\right\rangle\left|n^{-}\right\rangle-\left|m^{-}\right\rangle\left|n^{+}\right\rangle\right)
\end{aligned}
$$

Moreover, it is clear that, given that the states on $\alpha$ and $\beta$ are singlet states, for any factorizable unitary evolution the intermediate states will be of the form (7) for some $\hat{m}, \hat{n}$.

Example 2. Albert's example. Albert's example involves four spin- $\frac{1}{2}$ particles, initially in the state

$$
|\psi(\alpha)\rangle=\left|\Psi^{-}\right\rangle_{12}\left|\Psi^{-}\right\rangle_{34}
$$

At some time particles 1 and 3 pass by each other and exchange spin, and, at spacelike separation, particles 2 and 4 do the same. The two spin-swaps restore 
the original state, so a state history on which the two swaps occur simultaneously, the state remains unchanged for all time.

Though the system consists of four particles, the four particles do not remain at spacelike separation, and, so, to apply our result, which concerns systems that are spatially separated, we have to consider it as consisting of two subsystems, one consisting of particles 1 and 3, and the other consisting of particles 2 and 4 . Take $A$ to be the system consisting of particles 1 and 3 , and $B$ to be the system consisting of particles 2 and 4 . We can rewrite (8) as,

$$
\begin{aligned}
|\psi(\alpha)\rangle= & \frac{1}{2}\left(|+\rangle_{1}|-\rangle_{2}-|-\rangle_{1}|+\rangle_{2}\right)\left(|+\rangle_{3}|-\rangle_{4}-|-\rangle_{3}|+\rangle_{4}\right) \\
= & \frac{1}{2}\left(|+\rangle_{1}|+\rangle_{3} \otimes|-\rangle_{2}|-\rangle_{4}-|+\rangle_{1}|-\rangle_{3} \otimes|-\rangle_{2}|+\rangle_{4}\right. \\
& \left.-|-\rangle_{1}|+\rangle_{3} \otimes|+\rangle_{2}|-\rangle_{4}+|-\rangle_{1}|-\rangle_{3} \otimes|+\rangle_{2}|+\rangle_{4}\right) .
\end{aligned}
$$

Let

$$
\begin{array}{ll}
\left|a_{1}\right\rangle=|+\rangle_{1}|+\rangle_{3}, & \left|b_{1}\right\rangle=|-\rangle_{2}|-\rangle_{4}, \\
\left|a_{2}\right\rangle=|+\rangle_{1}|-\rangle_{3}, & \left|b_{2}\right\rangle=-|-\rangle_{2}|+\rangle_{4}, \\
\left|a_{3}\right\rangle=|-\rangle_{1}|+\rangle_{3}, & \left|b_{3}\right\rangle=-|+\rangle_{2}|-\rangle_{4}, \\
\left|a_{4}\right\rangle=|-\rangle_{1}|-\rangle_{3}, \quad\left|b_{4}\right\rangle=|+\rangle_{2}|+\rangle_{4} .
\end{array}
$$

Let $\left|a_{i}^{\prime}\right\rangle$ be the result of applying the the 1-3 spin swap to $\left|a_{i}\right\rangle$, and $\left|b_{i}^{\prime}\right\rangle$, the result of applying the 2-4 swap to $\left|b_{i}\right\rangle$.

$$
\begin{array}{ll}
\left|a_{1}^{\prime}\right\rangle=\left|a_{1}\right\rangle, \quad\left|b_{1}^{\prime}\right\rangle=\left|b_{1}\right\rangle, \\
\left|a_{2}^{\prime}\right\rangle=\left|a_{3}\right\rangle, \quad\left|b_{2}^{\prime}\right\rangle=\left|b_{3}\right\rangle, \\
\left|a_{3}^{\prime}\right\rangle=\left|a_{2}\right\rangle, \quad\left|b_{3}^{\prime}\right\rangle=\left|b_{2}\right\rangle, \\
\left|a_{4}^{\prime}\right\rangle=\left|a_{4}\right\rangle, \quad\left|b_{4}^{\prime}\right\rangle=\left|b_{4}\right\rangle,
\end{array}
$$

and let $c_{1}=-c_{2}=-c_{3}=c_{4}=\frac{1}{2}$. Then we have two Schmidt decompositions of the initial (and final) state):

$$
|\psi(\alpha)\rangle=|\psi(\beta)\rangle=\sum_{i=1}^{4} c_{i}\left|a_{i}\right\rangle\left|b_{i}\right\rangle=\sum_{i=1}^{4} c_{i}\left|a_{i}^{\prime}\right\rangle\left|b_{i}^{\prime}\right\rangle .
$$


The intermediate states are

$$
\begin{aligned}
& |\psi(\gamma)\rangle=\sum_{i} c_{i}\left|a_{i}^{\prime}\right\rangle\left|b_{i}\right\rangle \\
& |\psi(\delta)\rangle=\sum_{i} c_{i}\left|a_{i}\right\rangle\left|b_{i}^{\prime}\right\rangle
\end{aligned}
$$

Thus, Albert's example is an instance of our theorem.

\subsection{Non-isolated evolution}

We consider the case in which the systems are not isolated during the evolution from $\alpha$ to $\beta$, but interact with their environment. As mentioned above, we consider the causal past of $s_{A}(\beta)$ on $\alpha, \tilde{s}_{A}(\alpha)$, and the causal past of $s_{B}(\beta)$ on $\alpha$, $\tilde{s}_{B}(\alpha)$. There will be unitary operators $U_{A}, U_{B}$, such that

$$
\operatorname{Tr}[\rho(\beta) A \otimes B]=\operatorname{Tr}\left[\tilde{\rho}(\alpha) U_{A}^{\dagger} A U_{A} \otimes U_{B}^{\dagger} B U_{B}\right]
$$

for all $A \in \mathscr{A}, B \in \mathscr{B}$.

We consider first the case in which the state of $\tilde{s}_{A}(\alpha) \cup \tilde{s}_{B}(\alpha)$ is pure. Suppose that this state is represented by a state vector $|\tilde{\psi}(\alpha)\rangle$, with Schmidt representation

$$
|\tilde{\psi}(\alpha)\rangle=\sum_{i} c_{k}\left|\tilde{a}_{k}\right\rangle \otimes\left|\tilde{b}_{k}\right\rangle
$$

Let

$$
\left|a_{k}^{\prime}\right\rangle=U_{A}\left|\tilde{a}_{k}\right\rangle, \quad\left|b_{k}^{\prime}\right\rangle=U_{A}\left|\tilde{b}_{k}\right\rangle
$$

Let $V_{A}, V_{B}$ be unitary operators defined by

$$
\begin{aligned}
& V_{A}\left|\tilde{a}_{k}\right\rangle=e^{i \theta_{k}} U_{A}\left|\tilde{a}_{k}\right\rangle=e^{i \theta_{k}}\left|a_{k}^{\prime}\right\rangle \\
& V_{B}\left|\tilde{b}_{k}\right\rangle=e^{-i \theta_{k}} U_{B}\left|\tilde{b}_{k}\right\rangle=e^{-i \theta_{k}}\left|b_{k}^{\prime}\right\rangle .
\end{aligned}
$$

Then, obviously, $V_{A} \otimes V_{B}$ has the same effect on $|\tilde{\psi}(\alpha)\rangle$ as $U_{A} \otimes U_{B}$. Let $\rho(\gamma)$, $\rho^{\prime}(\gamma)$ be the states on $\gamma$ produced by evolving with $U_{A}$ and $V_{A}$, respectively. We will have,

$$
\begin{gathered}
\operatorname{Tr}[\rho(\gamma) A \otimes B]=\sum_{i, j} c_{i} c_{j}^{*}\left\langle a_{j}^{\prime}|A| a_{i}^{\prime}\right\rangle\left\langle\tilde{b}_{j}|B| \tilde{b}_{i}\right\rangle \\
\operatorname{Tr}\left[\rho^{\prime}(\gamma) A \otimes B\right]=\sum_{i, j} c_{i} c_{j}^{*} e^{i\left(\theta_{i}-\theta_{j}\right)}\left\langle a_{j}^{\prime}|A| a_{i}^{\prime}\right\rangle\left\langle\tilde{b}_{j}|B| \tilde{b}_{i}\right\rangle .
\end{gathered}
$$


for all $A \in \mathscr{A}, B \in \tilde{\mathscr{B}}$.

If, for all $A,\left\langle a_{j}^{\prime}|A| a_{i}^{\prime}\right\rangle=0$ for all distinct $i, j$, then

$$
\operatorname{Tr}[\rho(\beta) A \otimes B]=\sum_{i}\left|c_{i}\right|^{2}\left\langle a_{i}^{\prime}|A| a_{i}^{\prime}\right\rangle\left\langle b_{i}^{\prime}|B| b_{i}^{\prime}\right\rangle,
$$

for all $A \in \tilde{\mathscr{A}}, B \in \tilde{\mathscr{B}}$, and the evolution has disentangled the two systems. Suppose, now, that this is not the case, that there are distinct $i, j$, with $c_{i}, c_{j}$ nonzero, such that $\left\langle a_{i}^{\prime}|A| a_{j}^{\prime}\right\rangle$ is nonzero for some $A$. Choose

$$
B=\left|b_{j}\right\rangle\left\langle b_{i}|+| b_{i}\right\rangle\left\langle b_{j}\right|
$$

Then all we have to do is choose distinct $\theta_{i}, \theta_{j}$, to obtain

$$
\operatorname{Tr}[\rho(\gamma) A \otimes B] \neq \operatorname{Tr}\left[\rho^{\prime}(\gamma) A \otimes B\right]
$$

for some $A \in \mathscr{A}$.

Therefore, in the case of non-isolated evolution, we have non-narratability so long as the initial state of $\tilde{s}_{A}(\alpha) \cup \tilde{s}_{B}(\alpha)$ is entangled, and the evolution leaves $A$ and $B$ entangled.

\subsection{Systems of more than two components, pure initial states}

Now consider the case in which we have three or more systems, located in disjoint regions of space. We first consider the case in which the initial state of these systems is pure.

\subsubsection{Isolated evolution}

Take first the case of three systems, $A, B$, and $C$, spacelike separated from each other between $\alpha$ and $\beta$, and isolated from their environments. Then there will be unitary operators $U_{A}, U_{B}, U_{C}$, such that

$$
|\psi(\beta)\rangle=U_{A} \otimes U_{B} \otimes U_{C}|\psi(\alpha)\rangle .
$$

We ask whether there will exist unitaries $V_{A}, V_{B}, V_{C}$ such that

$$
|\psi(\beta)\rangle=V_{A} \otimes V_{B} \otimes V_{C}|\psi(\alpha)\rangle,
$$

but

$$
I \otimes I \otimes U_{C}|\psi(\alpha)\rangle \neq I \otimes I \otimes V_{C}|\psi(\alpha)\rangle .
$$


As we have seen in $\S 5.1 .1$, this is equivalent to asking whether there are unitaries $W_{A}, W_{B}, W_{C}$ such that $|\psi(\alpha)\rangle$ is invariant under $W_{A} \otimes W_{B} \otimes W_{C}$ but not under $I \otimes I \otimes W_{C}$.

Generically, $|\psi(\alpha)\rangle$ will be an entangled state. Consider the reduced state of $\rho_{A B}(\alpha)$ of $A B$. This will be an improper mixture, and we can write it as a mixture of mutually orthogonal pure states,

$$
\rho_{A B}(\alpha)=\sum_{i} w_{i} \delta_{i}
$$

If there are unitaries $W_{A}, W_{B}, W_{C}$ such that $|\psi(\alpha)\rangle$ is invariant under $W=W_{A} \otimes$ $W_{B} \otimes W_{C}$, then the action of $W_{A} \otimes W_{B}$ must leave the reduced state of $A+B$ invariant. Suppose, now, that there exist $w_{n}, w_{m}$ that differ from each other and all $w_{i}$, for $i \neq n, m$. Then we must have

$$
\begin{aligned}
W \delta_{n} W^{\dagger} & =\delta_{n} \\
W \delta_{m} W^{\dagger} & =\delta_{m}
\end{aligned}
$$

Suppose that the pure state $\delta_{n}$ is represented by a vector $\left|\psi_{n}\right\rangle$ whose Schmidt representation is non-degenerate. Then $W_{A} \otimes W_{B}$ can only change the relative phases of the terms of the Schmidt representations; the Schmidt bases for $A$ and $B$ must be eigenvectors of $U_{A}$ and $U_{B}$, respectively. But the same must be true for $\delta_{m}$, which means that the elements of the Schmidt bases for $\left|\psi_{m}\right\rangle$ must be parallel or orthogonal to the elements of the Schmidt bases $\left|\psi_{n}\right\rangle$.

This will be true only for very special states. It is easy to see that, if we have a state in which it holds can make them fail by perturbing $\delta_{n}$ slightly, whereas, if they fail, this will remain true under sufficiently small perturbations. Therefore, for generic improper mixtures $\rho_{A B}$, there will be no nontrivial factorizable unitary that leaves $\rho_{A B}$ invariant.

What this means is that, though, from the results of the previous section, there will always be distinct unitaries $U_{A B} \otimes U_{C}$ and $V_{A B} \otimes V_{C}$ such that

$$
U_{A B} \otimes U_{C}|\psi(\alpha)\rangle=V_{A B} \otimes V_{C}|\psi(\alpha)\rangle,
$$

we will not, in general, be able to satisfy the further requirement that $U_{A B}$ and $V_{A B}$ be factorizable, without sacrificing the desideratum that they yield different states when acting alone on $|\psi(\alpha)\rangle$.

For example, take the state $|\psi\rangle$ to be

$$
|\psi\rangle=c_{1}\left|\Phi_{1}\right\rangle_{A B}|+\rangle_{C}+c_{2}\left|\Phi_{2}\right\rangle_{A B}|-\rangle_{C}
$$


where $|+\rangle,|-\rangle$ are orthogonal vectors in $\mathscr{H}_{C}$, and $\left|\Phi_{1}\right\rangle_{A B},\left|\Phi_{2}\right\rangle_{A B}$ are orthogonal vectors in $\mathscr{H}_{A} \otimes \mathscr{H}_{B}$, and $c_{1} \neq c_{2}$. Then, as we know from Proposition 1 , a unitary operator of the form $W_{C}$ under which $|\psi\rangle$ is envariant can at best change the relative phases of the Schmidt decomposition of $|\psi\rangle$. That is, we will have

$$
U_{C}|+\rangle=e^{i \theta_{1}}|+\rangle \quad U_{C}|-\rangle=e^{i \theta_{2}}|-\rangle
$$

for some $\theta_{1}, \theta_{2}$. There can be no factorizable unitary $W_{A} \otimes W_{B}$ that undoes such a transformation unless $\left|\Phi_{1}\right\rangle_{A B}$ and $\left|\Phi_{2}\right\rangle_{A B}$ are distinguishable by local measurementsthat is, unless there exist observables $O_{A}$ and $O_{B}$ such that these states are distinct eigenstates of $O_{A} \otimes O_{B}$. This condition will be generically violated. Thus we have the following proposition.

Proposition 2 Let $\left|\psi_{\alpha}\right\rangle,\left|\psi_{\beta}\right\rangle\left|\psi_{\gamma}\right\rangle$ be vectors in a Hilbert space $\mathscr{H}_{A} \otimes \mathscr{H}_{B} \otimes \mathscr{H}_{C}$, related by,

$$
\begin{gathered}
\left|\psi_{\beta}\right\rangle=U_{A} \otimes U_{B} \otimes U_{C}\left|\psi_{\alpha}\right\rangle, \\
\left|\psi_{\gamma}\right\rangle=U_{A} \otimes U_{B} \otimes I_{C}\left|\psi_{\alpha}\right\rangle, \\
\left|\psi_{\delta}\right\rangle=I_{A} \otimes I_{B} \otimes U_{C}\left|\psi_{\alpha}\right\rangle .
\end{gathered}
$$

If

$$
\left|\psi_{\alpha}\right\rangle=\sum_{k} c_{k}\left|a_{k}\right\rangle_{A B}\left|b_{k}\right\rangle_{C}
$$

is a Schmidt decomposition of $\left|\psi_{\alpha}\right\rangle$ whose elements are not locally distinguishable, such that $\left|c_{n}\right| \neq\left|c_{i}\right|$ for $i \neq n$, then, if, for some $V_{A} V_{B}, V_{C}$, we have

$$
\left|\psi_{\beta}\right\rangle=V_{A} \otimes V_{B} \otimes V_{C}\left|\psi_{\alpha}\right\rangle,
$$

then

$$
V_{A} \otimes V_{B} \otimes I_{C}\left|\psi_{\alpha}\right\rangle \propto\left|\psi_{\gamma}\right\rangle
$$

and

$$
I_{A} \otimes I_{B} \otimes V_{C}\left|\psi_{\alpha}\right\rangle \propto\left|\psi_{\delta}\right\rangle
$$

\subsection{Mixed initial states}

Consider, now a bipartite system that is not initially in a pure state, but is somewhat entangled with its environment, and undergoes factorizable unitary evolution between hypersurfaces $\alpha$ and $\beta$. This is, essentially, the same as the situation considered in the previous section. As we saw, for generic improper mixtures $\rho_{A B}$, there will be no nontrivial factorizable unitary that leaves $\rho_{A B}$ invariant. 


\section{Conclusion}

The results obtained above show that, for systems consisting of spatially separated parts undergoing separate unitary evolution, non-narratability depends on very special initial states. For generic initial states, the state of the system on one hypersurface, together with the condition that evolutions of spacelike separated systems factorize, uniquely determines the state of the system on another hypersurface.

However, there is still an important difference between quantum state evolution in a relativistic spacetime and the evolution of classical states. For the latter, a state history along one foliation uniquely determines the state history along any other without any consideration of dynamics. In the quantum case, though the requirement that dynamics respect relativistic causality places strong constraints on possible state histories, dynamical considerations are indispensable for making the shift between state histories along different foliations.

\section{Acknowledgements}

This work was supported by a grant from the Foundational Questions Institute (FQXi).

\section{References}

Aharonov, Y. and D. Albert (1984). Is the usual notion of time evolution adequate for quantum-mechanical systems? II. Relativistic considerations. Physical Review $D$ 29, 228-234.

Albert, D. Z. (2015). Physics and narrative. In After Physics, pp. 106-123. Harvard University Press.

Albert, D. Z. and R. Galchen (2009, March). A quantum threat to special relativity. Scientific American 300, 32-39.

Myrvold, W. C. (2002). On peaceful coexistence: Is the collapse postulate incompatible with relativity? Studies in History and Philosophy of Modern Physics 33, 435-466. 
Zurek, W. H. (2005). Probabilities from entanglement, Born's rule $p_{k}=\left|\psi_{k}\right|^{2}$ from envariance. Physical Review A 71, 052105. 